\title{
Updating the Multipliers Associated with Inequality Constraints in an Augmented Lagrangian Multiplier Method
}

\author{
C. P. Avelino ${ }^{1}$ And L. N. Vicente ${ }^{2}$ \\ Communicated by Y. Zhang
}

\begin{abstract}
This paper contributes to the development of the field of augmented Lagrangian multiplier methods for general nonlinear programming by introducing a new update for the multipliers corresponding to inequality constraints. The update maintains naturally the nonnegativity of the multipliers without the need for a positive-orthant projection, as a result of the verification of the first-order necessary conditions for the minimization of a modified augmented Lagrangian penalty function.

In the new multiplier method, the roles of the multipliers are interchanged: the multipliers corresponding to the inequality constraints are updated explicitly, whereas the multipliers corresponding to the equality constraints are approximated implicitly. It is shown that the basic properties of local convergence of the traditional multiplier method are valid also for the proposed method.
\end{abstract}

Key Words. Nonlinear programming, multiplier methods, augmented Lagrangians, penalty functions, local convergence.

\section{Introduction}

We consider the general nonlinear programming problem, in the format

$$
\min f(x), \quad \text { s.t. } h(x)=0, x \geq 0,
$$

\footnotetext{
${ }^{1} \mathrm{PhD}$ Student, Departamento de Matemática, Universidade de Coimbra, Coimbra, Portugal. Support for this author was provided by Departamento de Matemática da UTAD, Centro de Matemática da Universidade de Coimbra, FCT Grant POCTI/35059/MAT/2000 and PRODEP 5.3 Program.

${ }^{2}$ Associate Professor, Departamento de Matemática, Universidade de Coimbra, Coimbra, Portugal. Support for this author was provided by Centro de Matemática da Universidade de Coimbra, FCT Grant POCTI/35059/MAT/2000, European Union Grant IST-2000-26063, and Fundação Calouste Gulbenkian.
} 
where $x \in \mathbb{R}^{n}$, the functions $f$ and $h$ are considered smooth and defined as $f: \Omega \rightarrow \mathbb{R}$ and $h: \Omega \rightarrow \mathbb{R}^{m}, n$ and $m$ are positive integers satisfying $n>m$, and $\Omega$ is an open set of $\mathbb{R}^{n}$.

The multiplier method (Refs. 1-2) is based on the augmented Lagrangian penalty function

$$
\begin{aligned}
L(x ; \lambda, \mu) & =f(x)+h(x)^{\top} \lambda+(1 / 2 \mu) h(x)^{\top} h(x) \\
& =l(x, \lambda)+(1 / 2 \mu) h(x)^{\top} h(x),
\end{aligned}
$$

where $\mu>0$ is a penalty parameter and

$$
l(x, \lambda)=f(x)+h(x)^{\top} \lambda
$$

is the Lagrangian of $f$ with respect to the equality constraints $h(x)=0$, with corresponding multipliers $\lambda \in \mathbb{R}^{m}$. Note that the Lagrangian term of the augmented Lagrangian penalty function involves only the equality constraints $h(x)=0$. In each outer iteration of this method, the primal iterate $x_{k}$ is computed by solving the problem

$$
\min L\left(x ; \lambda_{k}, \mu_{k}\right), \quad \text { s.t. } x \geq 0,
$$

for some $\lambda_{k}$ and $\mu_{k}>0$. The multiplier method (Ref. 1) updates the multipliers $\lambda$ for the next iteration by using the formula

$$
\lambda_{k+1}=\lambda_{k}+\left(1 / \mu_{k}\right) h\left(x_{k}\right) .
$$

Thus, the multipliers $\lambda$ corresponding to the equality constraints $h(x)=0$ are updated explicitly. The nonnegative multipliers $w \in \mathbb{R}^{n}$, corresponding to the inequality constraints $x \geq 0$ in problem (1), can be approximated implicitly from the multipliers associated with $x \geq 0$ in problem (2); see Ref. 2.

The question addressed in this paper is the interchange of the implicit vs. explicit roles of the multipliers in the multiplier method. It turns out that it is possible to derive a multiplier method where the multipliers $w$ corresponding to the inequality constraints are updated explicitly and kept nonnegative, whereas the multipliers corresponding to the equality constraints are approximated implicitly.

For this purpose, let us consider the duality part of the first-order necessary optimality conditions for problem (1),

$$
\nabla f(x)+\nabla h(x) \lambda-w=0,
$$

and the corresponding least-square Lagrange multiplier estimate [when $\nabla h(x)$ has full rank],

$$
\lambda(x, w)=-\left[\nabla h(x)^{\top} \nabla h(x)\right]^{-1} \nabla h(x)^{\top}[\nabla f(x)-w] .
$$


Therefore, it is possible to consider an augmented Lagrangian penalty function in the variables $x$, parameterized by the penalty parameter $\mu>0$ and the multiplier $w \geq 0$,

$$
P(x ; w, \mu)=f(x)+h(x)^{\top} \lambda(x, w)+(1 / 2 \mu) h(x)^{\top} h(x),
$$

and to pose the corresponding penalized problem,

$$
\min P(x ; w, \mu), \quad \text { s.t. } x \geq 0 .
$$

Each outer iteration of the new multiplier method involves the computation of the primal variables $x_{k}$ by solving the problem

$$
\min P\left(x ; w_{k}, \mu_{k}\right), \quad \text { s.t. } x \geq 0,
$$

for some $w_{k} \geq 0$ and $\mu_{k}>0$. Then, the outer iteration provides a formula to update $w$ for the next iteration,

$$
w_{k+1}=\nabla P\left(x_{k} ; w_{k}, \mu_{k}\right) .
$$

This formula results naturally from the first-order necessary conditions for problem (4) and guarantees the nonnegativity of the new multiplier estimate $w_{k+1}$.

In this paper, we establish the local convergence properties of the new multiplier method based on (4)-(5) for general programming problems of the form (1). Although the analysis presented here has a lot in common with the proof of local convergence for the original multiplier method (Ref. 1), several difficulties inherent to the nature of the new update had to be overcome. In particular, it is shown that the neighborhood of local convergence is smaller than in the original multiplier method [see (13)]. The new multiplier method was developed originally in Ref. 3 for nonlinear optimization problems of the form

$$
\min f(y, u), \quad \text { s.t. } c(y, u)=0,(y, u) \geq 0,
$$

where it was assumed that the partial Jacobian of $c$ with respect to $y$ is square and invertible.

The paper is structured as follows. In Section 2, we describe the new multiplier method for (1) in more detail. Then, the local convergence properties are presented in Section 3. In Section 4, we state some conclusions and comments. The proof of the main result of the local convergence analysis, stated in Theorem 3.2, is given in the Appendix of the paper (Section 5). 


\section{New Multiplier Method}

A point $x$ satisfies the first-order necessary optimality conditions for problem (1) if there exist $\lambda \in \mathbb{R}^{m}$ and $w \in \mathbb{R}^{n}$ such that

$$
\begin{aligned}
& \nabla_{x} l(x, \lambda)-w=0, \\
& h(x)=0, \quad x \geq 0, \\
& x^{\top} w=0, \quad w \geq 0 .
\end{aligned}
$$

Conditions (7) are know as the first-order Karush-Kuhn-Tucker conditions and can be written in the equivalent form

$$
\begin{aligned}
& Z(x)^{\top}(\nabla f(x)-w)=0, \\
& h(x)=0, \quad x \geq 0, \\
& x^{\top} w=0, \quad w \geq 0,
\end{aligned}
$$

where $Z(x)$ is a matrix whose columns form an orthonormal basis for the null space of $\nabla h(x)^{\top}$; i.e., $Z(x)$ satisfies

$$
Z(x)^{\top} Z(x)=I \text { and } \nabla h(x)^{\top} Z(x)=0 .
$$

The matrix $Z(x)$ can be obtained from the QR factorization of $\nabla h(x)$.

Note that the matrix

$$
Z(x) Z(x)^{\top}=I-\nabla h(x)\left[\nabla h(x)^{\top} \nabla h(x)\right]^{-1} \nabla h(x)^{\top}
$$

is an orthogonal projector onto the null space $\mathscr{N}\left(\nabla h(x)^{\top}\right)$ of the matrix $\nabla h(x)^{\top}$. Similarly, $I-Z(x) Z(x)^{\top}$ is an orthogonal projector onto $\mathscr{R}(\nabla h(x))$, the range space of $\nabla h(x)$.

The first-order and second-order derivatives of the penalty function $P$ require the second-order and third-order derivatives of $f$ and $h$, respectively. Therefore, to establish the local convergence properties, we will need the following assumptions that will be kept throughout this paper.

(A1) The functions $f$ and $h$ are three times continuously differentiable in $\Omega$, where $\Omega$ is an open set of $\mathbb{R}^{n}$. The Jacobian matrix $\nabla h(x)^{\top}$ of $h(x)$ has full rank in $\Omega$.

We point out that an implementation of the multiplier method (with or without a globalization scheme) could require only first-order or secondorder derivatives.

To derive the first-order necessary conditions for problem (3), we need first to calculate the gradient of $P(x ; w, \mu)$ with respect to $x$. First, we note 
that

$$
\nabla_{x} \lambda(x, w)=-\left[\nabla_{x x}^{2} l(x, \lambda(x, w)) \nabla h(x)+R(x, w)^{\top}\right]\left[\nabla h(x)^{\top} \nabla h(x)\right]^{-1},
$$

where the $i$ th row of $R(x, w)$ is given by

$$
R(x, w)_{i}=\left[\nabla_{x} l(x, \lambda(x, w))-w\right]^{\top} \nabla^{2} h_{i}(x), \quad i=1, \ldots, m .
$$

Thus, the gradient of $P(x ; w, \mu)$ is given by

$$
\nabla P(x ; w, \mu)=G_{1}(x ; w, \mu)+G_{2}(x ; w, \mu)+G_{3}(x ; w, \mu),
$$

where

$$
\begin{aligned}
& G_{1}(x ; w, \mu)=Z(x) Z(x)^{\top} \nabla f(x)+\left[I-Z(x) Z(x)^{\top}\right] w, \\
& G_{2}(x ; w, \mu)=-\left[\nabla_{x x}^{2} l(x, \lambda(x, w)) \nabla h(x)+R(x, w)^{\top}\right]\left[\nabla h(x)^{\top} \nabla h(x)\right]^{-1} h(x), \\
& G_{3}(x ; w, \mu)=(1 / \mu) \nabla h(x) h(x) .
\end{aligned}
$$

To simplify the notation, we will omit the arguments $x$ and $x^{*}$ when it is clear from the context where the functions are evaluated. For instance,

$$
\nabla h=\nabla h(x) \text { and } \nabla f^{*}=\nabla f\left(x^{*}\right) .
$$

A point $x$ satisfies the first-order necessary conditions for problem (3) if there exists $\bar{w} \in \mathbb{R}^{n}$ such that

$$
\begin{aligned}
& Z Z^{\top} \nabla f+\left(I-Z Z^{\top}\right) w-\left(\nabla_{x x}^{2} l \nabla h+R^{\top}\right)\left(\nabla h^{\top} \nabla h\right)^{-1} h \\
& +(1 / \mu) \nabla h h-\bar{w}=0, \\
& x \geq 0, \\
& x^{\top} \bar{w}=0, \quad \bar{w} \geq 0 .
\end{aligned}
$$

Equation (9a) provides an update formula for the multipliers corresponding to the constraints $x \geq 0$, that is the basis of the multiplier method considered in this paper.

The penalty function $P$, together with the penalized problem (3) and the equation (9a), suggest a new multiplier method to solve the nonlinear programming problem (1), which is presented below without any globalization strategy.

\section{Algorithm 2.1.}

Step 0. Choose initial values $\mu_{0}$ for the penalty parameter and $w_{0}$ for the approximation of the multipliers.

Step 1. For $k=0,1,2, \ldots$, do the following steps. 
Step 1a. Solve problem (4).

Step 1b. Update the multipliers approximation,

$$
\begin{aligned}
w_{k+1}= & Z Z^{\top} \nabla f+\left(I-Z Z^{\top}\right) w_{k} \\
& -\left(\nabla_{x x}^{2} l \nabla h+R^{\top}\right)\left(\nabla h^{\top} \nabla h\right)^{-1} h+\left(1 / \mu_{k}\right) \nabla h h,
\end{aligned}
$$

where the functions $\nabla f, h, \nabla h, Z$ are evaluated at the solution $\tilde{x}\left(w_{k}, \mu_{k}\right)$ obtained in Step 1a and the functions $\nabla_{x x}^{2} l, R$ are evaluated at $\left(\tilde{x}\left(w_{k}, \mu_{k}\right), \lambda\left(\tilde{x}\left(w_{k}, \mu_{k}\right), w_{k}\right)\right)$.

Step 1c. Update the penalty parameter $\mu_{k+1}$.

The local convergence analysis of the multiplier method, based on Algorithm 2.1, is presented in Section 3 and corresponds to the analysis given in Bertsekas (Ref. 1) for the traditional augmented Lagrangian multiplier method.

\section{Local Convergence Analysis}

The study of the rate of local convergence of the multiplier method (as described in Algorithm 2.1) requires the second derivatives of the penalty function $P(x ; w, \mu)$. One can show easily that the Hessian matrix of $P(x ; w, \mu)$ is given by

$$
\nabla^{2} P(x ; w, \mu)=H_{1}(x ; w, \mu)+H_{2}(x ; w, \mu)+H_{3}(x ; w, \mu),
$$

where the matrices

$$
\begin{aligned}
& H_{1}(x ; w, \mu)=\nabla_{x x}^{2} l Z Z^{\top}-R^{\top}\left(\nabla h^{\top} \nabla h\right)^{-1} \nabla h^{\top}, \\
& H_{2}(x ; w, \mu)=-\left(I-Z Z^{\top}\right) \nabla_{x x}^{2} l-\nabla h\left(\nabla h^{\top} \nabla h\right)^{-1} R+\sum_{i=1}^{m} h_{i} \nabla_{x x}^{2} \lambda_{i}, \\
& H_{3}(x ; w, \mu)=(1 / \mu) \nabla h \nabla h^{\top}+(1 / \mu) \sum_{i=1}^{m} h_{i} \nabla^{2} h_{i},
\end{aligned}
$$

correspond to the derivatives of $G_{1}(x ; w, \mu), G_{2}(x ; w, \mu), G_{3}(x ; w, \mu)$ in (8), respectively.

We start by showing that the penalty function $P(x ; w, \mu)$ exhibits some exactness properties. The result stated in the next theorem will be helpful later in the analysis of the local convergence, in particular the fact that the Hessian of $P(x ; w, \mu)$ is positive definite for $\mu$ in $\left(0, \mu^{*}\right]$, where $\mu^{*}>0$ is specified later, provided that $x$ satisfies the second-order sufficient conditions for the original problem (1) with multipliers $\lambda(x, w)$ and $w$. 
Theorem 3.1. Let Assumptions (A1) hold. If $(x, \lambda(x, w))$ satisfies the second-order necessary [resp. sufficient] conditions for the original problem (1), with multipliers $w$ corresponding to $x \geq 0$, then there exists $\mu^{*}>0$ such that $x$ satisfies the second-order necessary [resp. sufficient] conditions for the penalized problem (3), for this $w$ and for any $\mu \in\left(0, \mu^{*}\right]$.

Proof. We start by pointing out that, because the matrix

$$
\left[\begin{array}{l}
Z(x)^{\top} \\
\nabla h(x)^{\top}
\end{array}\right]
$$

is nonsingular, equation (9a), when $h(x)=0$, is equivalent to

$$
Z(x)^{\top}[\nabla f(x)-\bar{w}]=0 \text { and } \nabla h(x)^{\top}(w-\bar{w})=0 .
$$

Thus, from the fact that $x$ satisfies the first-order necessary conditions for the original problem (1) with multipliers $\lambda(x, w)$ and $w$, we conclude that $x$ satisfies also the first-order necessary conditions (9) for the penalized problem (3) with multipliers $\bar{w}=w$.

Now, let us prove the result concerning the second-order sufficient conditions. For this purpose, let $\Delta x$ satisfy

$$
\begin{array}{ll}
(\Delta x)_{i}=0, & \text { if } x_{i}=0 \text { and } \bar{w}_{i}>0, \\
(\Delta x)_{i} \geq 0, & \text { if } x_{i}=0 \text { and } \bar{w}_{i}=0 .
\end{array}
$$

Since $h(x)=0$ and $R(x, w)=0$, we have

$$
\begin{aligned}
\Delta x^{\top} \nabla^{2} P(x ; w, \mu) \Delta x & \\
= & \Delta x^{\top} Z Z^{\top} \nabla_{x x}^{2} l Z Z^{\top} \Delta x-\Delta x^{\top}\left(I-Z Z^{\top}\right) \nabla_{x x}^{2} l\left(I-Z Z^{\top}\right) \Delta x \\
& +(1 / \mu) \Delta x^{\top} \nabla h \nabla h^{\top} \Delta x .
\end{aligned}
$$

On the other hand, the second-order sufficient conditions for the original problem (1) say that $\nabla_{x x}^{2} l(x, \lambda(x, w))$ has to be positive definite for all vectors $\Delta x$ satisfying (11) and

$$
\nabla h(x)^{\top} \Delta x=0,
$$

i.e.,

$$
\Delta x=Z Z^{\top} \Delta x .
$$

Thus,

$$
\Delta x^{\top} Z Z^{\top} \nabla_{x x}^{2} l Z Z^{\top} \Delta x>0,
$$

for all vectors $\Delta x$ satisfying (11).

So, since 


$$
I-Z Z^{\top}=\nabla h\left(\nabla h^{\top} \nabla h\right)^{-1} \nabla h^{\top},
$$

we have

$$
\begin{aligned}
& \Delta x^{\top} \nabla^{2} P(x ; w, \mu) \Delta x \\
& >\Delta x^{\top} \nabla h\left[-\left(\nabla h^{\top} \nabla h\right)^{-1} \nabla h^{\top} \nabla_{x x}^{2} l \nabla h\left(\nabla h^{\top} \nabla h\right)^{-1}+(1 / \mu) I\right] \nabla h^{\top} \Delta x,
\end{aligned}
$$

and the proof is completed by setting

$$
\mu^{*}= \begin{cases}\text { any positive real, } & \text { if }\left(\nabla h^{\top} \nabla h\right)^{-1} \nabla h^{\top} \nabla_{x x}^{2} l \nabla h\left(\nabla h^{\top} \nabla h\right)^{-1} \\ 1 / \alpha(x, w), & \text { is negative semidefinite, } \\ \text { otherwise, }\end{cases}
$$

where $\alpha(x, w)$ is the largest eigenvalue of the matrix

$$
\left(\nabla h^{\top} \nabla h\right)^{-1} \nabla h^{\top} \nabla_{x x}^{2} l \nabla h\left(\nabla h^{\top} \nabla h\right)^{-1} .
$$

The local convergence properties of the multiplier method are established under assumptions (A1) and (A2), where (A2) is given below.

(A2) The point $x^{*} \in \Omega$ is a nondegenerate point (i.e., the gradients of the functions defining the active constraints are linearly independent) satisfying the second-order sufficient conditions for problem (1) with corresponding multipliers $\lambda\left(x^{*}, \bar{w}^{*}\right)$ and $\bar{w}^{*}$. The pair $\left(x^{*}, \bar{w}^{*}\right)$ satisfies strict complementarity.

The main result is presented in Theorem 3.2 and bounds the distance between a local minimizer of (3) and $\left(x^{*}, \bar{w}^{*}\right)$ by the penalty parameter $\mu$ times the distance between the approximation $w$ and the corresponding multipliers $\bar{w}^{*}$. The proof of this theorem is quite long and technical and is postponed to the appendix of this paper.

Theorem 3.2. Let $x^{*}$, with corresponding multipliers $\bar{w}^{*}$, satisfy Assumptions (A1)-(A2). There exist positive scalars $\bar{\mu}, \delta, \epsilon, \kappa_{1}, \kappa_{2}$ such that the matrix

$$
Z^{*} Z^{* \top} \nabla_{x x}^{2} l^{*} Z^{*} Z^{*^{\top}}-\left(I-Z^{*} Z^{*^{\top}}\right) \nabla_{x x}^{2} l^{*}\left(I-Z^{*} Z^{*^{\top}}\right)+(1 / \bar{\mu}) \nabla h^{*} \nabla h^{* \top}
$$

is positive definite, the problem

$$
\min P(x ; w, \mu), \quad \text { s.t. } x \geq 0, x \in B\left(x^{*} ; \epsilon\right),
$$

has a unique solution $\tilde{x}(w, \mu)$ for all $(w, \mu)$ in

$$
D^{*}=\left\{(w, \mu):\left\|w-\bar{w}^{*}\right\|<\min \{\delta, \delta / \mu\} ; 0<\mu \leq \bar{\mu}\right\},
$$


the function $\tilde{x}(w, \mu)$ is continuously differentiable in $D^{*}$ and, for all $(w, \mu) \in D^{*}$, we have

$$
\begin{aligned}
& \left\|\tilde{x}(w, \mu)-x^{*}\right\| \leq \kappa_{1} \mu\left\|w-\bar{w}^{*}\right\|, \\
& \left\|\tilde{w}(w, \mu)-\bar{w}^{*}\right\| \leq \kappa_{2} \mu\left\|w-\bar{w}^{*}\right\|,
\end{aligned}
$$

where $\tilde{w}=\tilde{w}(w, \mu)$ are the multipliers corresponding to $\tilde{x}=\tilde{x}(w, \mu)$ and

$$
\begin{aligned}
\tilde{w}= & Z(\tilde{x}) Z(\tilde{x})^{\top} \nabla f(\tilde{x})+\left[I-Z(\tilde{x}) Z(\tilde{x})^{\top}\right] w \\
& -\left[\nabla_{x x}^{2} l(\tilde{x}, w) \nabla h(\tilde{x})+R(\tilde{x}, w)^{\top}\right]\left[\nabla h(\tilde{x})^{\top} \nabla h(\tilde{x})\right]^{-1} h(\tilde{x}) \\
& +(1 / \mu) \nabla h(\tilde{x}) h(\tilde{x}) .
\end{aligned}
$$

Theorem 3.2 can be used to state the basic local convergence properties of the multiplier method given in Algorithm 2.1, which we summarize in the next corollary.

Corollary 3.1. Let $x^{*}$, with corresponding multipliers $\bar{w}^{*}$, satisfy Assumptions (A1)-(A2). There exist scalars $\delta_{0} \in(0, \delta], \kappa \in(0,1), \mu_{0} \in(0, \bar{\mu}]$ such that, if the sequence $\left\{\mu_{k}\right\}$ is monotone decreasing and $\left\|w_{0}-\bar{w}^{*}\right\|<\min \left\{\delta_{0}, \delta_{0} / \mu_{0}\right\}$, then the sequence $\left\{w_{k}\right\}$, generated by $w_{k+1}=\nabla P\left(\tilde{x}\left(w_{k}, \mu_{k}\right) ; w_{k}, \mu_{k}\right)$, is well defined (in the sense that $\left(w_{k}, \mu_{k}\right) \in D^{*}$ for all $k$ ) and satisfies

$$
\limsup _{k \rightarrow+\infty}\left\|w_{k+1}-\bar{w}^{*}\right\| /\left\|w_{k}-\bar{w}^{*}\right\| \leq \kappa, \quad \text { when } \lim _{k \rightarrow+\infty} \mu_{k}>0,
$$

and

$$
\lim _{k \rightarrow+\infty}\left\|w_{k+1}-\bar{w}^{*}\right\| /\left\|w_{k}-\bar{w}^{*}\right\|=0, \quad \text { when } \lim _{k \rightarrow+\infty} \mu_{k}=0 .
$$

In both cases, we have

$$
\begin{aligned}
& \lim _{k \rightarrow+\infty} \tilde{x}\left(w_{k}, \mu_{k}\right)=x^{*}, \\
& \lim _{k \rightarrow+\infty} w_{k}=\bar{w}^{*} .
\end{aligned}
$$

Proof. The limits (16), (17), (19) follow from inequality (14b). The limit (18) is a consequence of (14a).

It is also worthwhile to note that the multipliers update (15) can be seen as an approximation to the steepest ascent iteration applied to the dual function associated with problem (12); see Ref. 3 for details on how this was carried out in the context of problem (6). 


\section{Conclusions and Future Research}

The augmented Lagrangian multiplier method proposed in this paper is based on the solution of a sequence of bound-constrained minimization problems. Each outer iteration of the method involves the minimization, within the bounds, of the augmented Lagrangian penalty function $P(x ; w, \mu)$ for specific values of the penalty parameter $\mu$ and the multipliers $w$. The evaluation of $P(x ; w, \mu)$ and its gradient requires the solution of systems of linear equations with $\nabla h(x)^{\top} \nabla h(x)$. The gradient of $P(x ; w, \mu)$ involves a cross term where second-order derivatives of the problem functions $f$ and $h$ appear. Thus, each inner or minor iteration [i.e., each iteration of the iterative process applied to minimize $P(x ; w, \mu)$ within the bounds] is relatively costly.

This augmented Lagrangian multiplier method was proposed originally in Ref. 3 for a class of nonlinear programming problems with a structure arising from optimal control or design [see (6)]. There, the role of the matrix $\nabla h(x)^{\top} \nabla h(x)$ is played by the matrix $c_{y}(y, u)$, the partial Jacobian of $c(y, u)$ with respect to the state variables $y$. There, the computation of the gradient of the penalty function involves the solution of linear systems with $c_{y}(y, u)$ (linearized state equations) and with $c_{y}(y, u)^{\top}$ (adjoint equations), for which solvers are available in many applications; see Ref. 4.

One major open question is weather a globalization scheme, similar to what was developed in Ref. 2 for the original multiplier method, would be applicable to the new multiplier method of this paper, yielding the same type of global convergence. In contrast to what happens in Ref. 2, we do not have here the equality

$$
\nabla P(x ; w, \mu)=\nabla_{x} l(x, \lambda(x, \bar{w}))
$$

that seems to us to be crucial to the derivation of global convergence. Instead, what we get is the following:

$$
\begin{aligned}
& \nabla P(x ; w, \mu)-\nabla_{x} l(x, \lambda(x, \bar{w})) \\
& =-Z(x) Z(x)^{\top}\left[\nabla_{x x}^{2} l(x, \lambda(x, w)) \nabla h(x)+R(x, w)^{\top}\right]\left[\nabla h(x)^{\top} \nabla h(x)\right]^{-1} h(x) .
\end{aligned}
$$

Of course, when $h(x)=0$, we do have

$$
\nabla P(x ; w, \mu)=\nabla_{x} l(x, \lambda(x, \bar{w})) .
$$

The fact that there is a term depending on the size of the feasibility function $h(x)$ in (20) makes the global analysis considerably more difficult.

Numerical results obtained for problems of small-scale dimension have shown that the method is competitive with Lancelot (Ref. 5), sharing some 
of the advantages and disadvantages of the class of augmented Lagrangian multiplier methods.

\section{Appendix: Proof of the Main Result}

We prove here the main result of local convergence established in Theorem 3.2. We will use the following notation. The symbol $e$ represents a vector of ones with appropriate size and $e_{i}$ denotes a vector whose $i$ th component is unity and the other components are zero. Also, for any vector $v, V$ is the diagonal matrix for which the diagonal elements are the elements of $v$.

Although the structure of the proof follows the one in Ref. 1, Proposition 2.4, we have additional difficulties here due to the presence of the bound constraints on the variables. Another difficulty arises when dealing with the cross term in the multipliers update. This term is not multiplied by $1 / \mu_{k}$ but involves $w_{k}$. A consequence of having to handle this extra term is that the region $D^{*}$ in (13) becomes smaller than the one in Ref. 1, Proposition 2.4, where instead of $\min \{\delta, \delta / \mu\}$ we only have $\delta / \mu$.

We need first to organize some of the calculations that will appear later. The derivative of

$$
s(x)=\left[\nabla h(x)^{\top} \nabla h(x)\right]^{-1} h(x)
$$

is given by

$$
\begin{aligned}
\nabla s(x)^{\top} & =\left(\nabla h^{\top} \nabla h\right)^{-1} \nabla h^{\top}-\left(\nabla h^{\top} \nabla h\right)^{-1} \sum_{i=1}^{m} \nabla\left(\nabla h^{\top} \nabla h\right)_{i}\left[\left(\nabla h^{\top} \nabla h\right)^{-1} h\right]_{i} \\
& \stackrel{\text { def }}{=}\left(\nabla h^{\top} \nabla h\right)^{-1} \nabla h^{\top}-F(h),
\end{aligned}
$$

where we have omitted the argument $x$ in the right-hand side. The size of $F(h(x))$ varies continuously with $h(x)$.

Further, we note that, from

$$
\nabla h(x)^{\top} Z(x)=0,
$$

one obtains

$$
\nabla h(x)^{\top} \nabla Z(x)_{j}^{\top}=-\left[\begin{array}{c}
Z(x)_{j}^{\top} \nabla^{2} h(x)_{1} \\
\vdots \\
Z(x)_{j}^{\top} \nabla^{2} h(x)_{m}
\end{array}\right],
$$


for $j=1, \ldots, n-m$, where $Z(x)_{j}$ denotes the $j$ th column of $Z(x)$. By using (21), we can write

$$
\left[\begin{array}{c}
(\nabla f-w)^{\top}\left(I-Z Z^{\top}\right) \nabla Z_{1}^{\top} \\
\vdots \\
(\nabla f-w)^{\top}\left(I-Z Z^{\top}\right) \nabla Z_{n-m}^{\top}
\end{array}\right]=-Z^{\top} \sum_{i=1}^{m}\left[\left(\nabla h^{\top} \nabla h\right)^{-1} \nabla h^{\top}(\nabla f-w)\right]_{i} \nabla^{2} h_{i} .
$$

We have assumed that $Z(x)$ is differentiable. Goodman (Ref. 6) has shown how to extend locally an orthonormal basis $Z(x)$ given by the QR factorization of $\nabla h(x)$ so that $Z(x)$ exhibits the same smoothness of $h(x)$.

Finally, we get an expression that will be used later on,

$$
\left[\begin{array}{c}
(\nabla f-w)^{\top} \nabla Z_{1}^{\top} \\
\vdots \\
(\nabla f-w)^{\top} \nabla Z_{n-m}^{\top}
\end{array}\right]=\left[\begin{array}{c}
(\nabla f-w)^{\top} Z Z^{\top} \nabla Z_{1}^{\top} \\
\vdots \\
(\nabla f-w)^{\top} Z Z^{\top} \nabla Z_{n-m}^{\top}
\end{array}\right]-Z^{\top} \nabla^{2} f+Z^{\top} \nabla_{x x}^{2} l .
$$

Now, we are ready to prove Theorem 3.2. The proof is divided in six major steps.

\section{Proof of Theorem 3.2}

Step 1. Preparing the System of Nonlinear Equations. For $\mu>0$, consider the system of nonlinear equations that results from the first-order necessary conditions (9) for problem (3). If we multiply equation (9a) by $\nabla h^{\top}$ and $Z^{\top}$, we obtain the equivalent system

$$
\begin{aligned}
& \nabla h^{\top} w-\nabla h^{\top}\left[\nabla_{x x}^{2} l(x, w) \nabla h+R(x, w)^{\top}\right]\left(\nabla h^{\top} \nabla h\right)^{-1} h \\
& +(1 / \mu) \nabla h^{\top} \nabla h h-\nabla h^{\top} \bar{w}=0, \\
& Z^{\top} \nabla f-Z^{\top}\left[\nabla_{x x}^{2} l(x, w) \nabla h+R(x, w)^{\top}\right]\left(\nabla h^{\top} \nabla h\right)^{-1} h-Z^{\top} \bar{w}=0, \\
& X \bar{W} e=0 .
\end{aligned}
$$

Now, we multiply equation (23a) by $\mu$ and perform the changes of variables

$$
\begin{aligned}
& r=\mu\left(w-\bar{w}^{*}\right), \\
& s=w-\bar{w}^{*},
\end{aligned}
$$

to obtain the system of nonlinear equations

$\nabla h^{\top} r-\mu \nabla h^{\top}\left[\nabla_{x x}^{2} l\left(x, \bar{w}^{*}+s\right) \nabla h+R\left(x, \bar{w}^{*}+s\right)^{\top}\right]\left(\nabla h^{\top} \nabla h\right)^{-1} h$

$+\nabla h^{\top} \nabla h h+\mu \nabla h^{\top} \bar{w}^{*}-\mu \nabla h^{\top} \bar{w}=0$,

$Z^{\top} \nabla f-Z^{\top}\left[\nabla_{x x}^{2} l\left(x, \bar{w}^{*}+s\right) \nabla h+R\left(x, \bar{w}^{*}+s\right)^{\top}\right]\left(\nabla h^{\top} \nabla h\right)^{-1} h-Z^{\top} \bar{w}=0$, $X \bar{W} e=0$, 
that we write as

$$
J(x, w, \mu)=0 .
$$

We analyze this system for $\mu \in\left[0, \mu^{*}\right]$, where $\mu^{*}$ is such that the matrix

$\mu Z^{*} Z^{* \top} \nabla_{x x}^{2} l^{*} Z^{*} Z^{* \top}-\mu\left(I-Z^{*} Z^{* \top}\right) \nabla_{x x}^{2} l^{*}\left(I-Z^{*} Z^{*^{\top}}\right)+\nabla h^{*} \nabla h^{*^{\top}}$

is positive definite for all $\mu \in\left(0, \mu^{*}\right]$. The existence of such $\mu^{*}>0$ is guaranteed by Theorem 3.1 .

Step 2. Nonsingularity at the Solution When the Penalty Parameter Is Zero. When $r=s=0$ and $\mu \in\left[0, \mu^{*}\right]$, it is easy to check that the system (25) has the solution $\left(x^{*}, \bar{w}^{*}\right)$. For $r=s=0$, the Jacobian of (25) with respect to $(x, \bar{w})$, at the point $\left(x^{*}, \bar{w}^{*}\right)$ is given by

$J^{*}(0,0, \mu)=\left[\begin{array}{lc}-\mu \nabla h^{* \top} \nabla_{x x}^{2} l^{*}\left(I-Z^{*} Z^{* \top}\right)+\nabla h^{* \top} \nabla h^{*} \nabla h^{* \top} & -\mu \nabla h^{* \top} \\ Z^{* \top} \nabla_{x x}^{2} l^{*} Z^{*} Z^{* \top} & -Z^{* \top} \\ \bar{W}^{*} & X^{*}\end{array}\right]$.

When $\mu=0, J^{*}(0,0, \mu)$ reduces to

$$
J^{*}(0,0,0)=\left[\begin{array}{lc}
\nabla h^{* \top} \nabla h^{*} \nabla h^{* \top} & 0 \\
Z^{* \top} \nabla_{x x}^{2} l^{*} Z^{*} Z^{* \top} & -Z^{* \top} \\
\bar{W}^{*} & X^{*}
\end{array}\right] .
$$

One can see that $J^{*}(0,0,0)$ is nonsingular. In fact, the assumptions on $\left(x^{*}, \bar{w}^{*}\right)$ imply that the following matrix is nonsingular:

$$
\left[\begin{array}{llc}
\nabla h^{* \top} & 0 & 0 \\
Z^{*} Z^{* \top} \nabla_{x x}^{2} l^{*} Z^{*} Z^{* \top} & \nabla h^{*} & -I \\
\bar{W}^{*} & 0 & X^{*}
\end{array}\right]
$$

The nonsingularity of (28) implies the nonsingularity of (27).

Step 3. Nonsingularity at the Solution for Positive Values of the Penalty Parameter. Let $(\Delta x, \Delta w)$ be a solution of the following homogeneous linear system with the matrix $J^{*}(0,0, \mu)$ :

$$
\begin{aligned}
& {\left[-\mu \nabla h^{* \top} \nabla_{x x}^{2} l^{*}\left(I-Z^{*} Z^{*^{\top}}\right)+\nabla h^{* \top} \nabla h^{*} \nabla h^{*^{\top}}\right] \Delta x-\mu \nabla h^{*^{\top}} \Delta w=0,} \\
& Z^{*^{\top}} \nabla_{x x}^{2} l^{*} Z^{*} Z^{*^{\top}} \Delta x-Z^{* \top} \Delta w=0, \\
& \bar{W}^{*} \Delta x+X^{*} \Delta w=0 .
\end{aligned}
$$

Equation (29c) and strict complementarity between $x^{*}$ and $\bar{w}^{*}$ imply

$$
\Delta x^{\top} \Delta w=0 .
$$


By multiplying (29a) and (29b) on the left by $\nabla h^{*}\left(\nabla h^{* \top} \nabla h^{*}\right)^{-1}$ and $\mu Z^{*}$, respectively, we obtain

$\left[-\mu\left(I-Z^{*} Z^{* \top}\right) \nabla_{x x}^{2} *^{*}\left(I-Z^{*} Z^{* \top}\right)+\nabla h^{*} \nabla h^{* \top}\right] \Delta x-\mu\left(I-Z^{*} Z^{* \top}\right) \Delta w=0$, $\mu Z^{*} Z^{* \top} \nabla_{x x}^{2}{ }^{*} Z^{*} Z^{* \top} \Delta x-\mu Z^{*} Z^{* \top} \Delta w=0$.

Thus,

$$
\begin{aligned}
& \mu Z^{*} Z^{* \top} \nabla_{x x}^{2} l^{*} Z^{*} Z^{* \top} \Delta x-\mu\left(I-Z^{*} Z^{* \top}\right) \nabla_{x x}^{2} l^{*}\left(I-Z^{*} Z^{* \top}\right) \Delta x \\
& +\nabla h^{*} \nabla h^{* \top} \Delta x-\mu \Delta w=0 .
\end{aligned}
$$

By multiplying this equation on the left by $\Delta x^{\top}$, we obtain

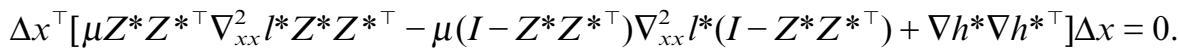

Since (26) is positive definite for $\mu \in\left(0, \mu^{*}\right]$, we conclude that

$$
\Delta x=0 .
$$

Now, using $\Delta x=0$, we get

$$
\nabla h^{*^{\top}} \Delta w=0 \text { and } Z^{*^{\top} \Delta w}=0,
$$

implying that

$$
\Delta w=0 .
$$

Therefore, we have proved that $J^{*}(0,0, \mu)$ is nonsingular for $\mu \in\left(0, \mu^{*}\right]$.

Step 4. Use of the Implicit Function Theorem. Now, we apply the implicit function theorem (Ref. 1, page 12) to the system (25). By identifying the set

$$
K=\{0\} \times\{0\} \times\left[0, \mu^{*}\right]
$$

as the compact set $\bar{X}$ of that theorem, we guarantee the existence of positive scalars $\epsilon$ and $\delta$ and unique continuously differentiable functions

$$
\hat{x}=\hat{x}(r, s, \mu) \quad \text { and } \quad \hat{w}=\hat{w}(r, s, \mu),
$$

defined on a neighborhood of $K$, $B(K, \delta)=\left\{(r, s, \mu):\left\|(r, s, \mu)-\left(0,0, \mu^{\prime}\right)\right\|<\delta\right.$, for some $\left.\left(0,0, \mu^{\prime}\right) \in K\right\}$, satisfying (25), with

$$
x=\hat{x}=\hat{x}(r, s, \mu) \quad \text { and } \quad \bar{w}=\hat{w}=\hat{w}(r, s, \mu),
$$

and such that

$$
\left\|\left[\begin{array}{l}
\hat{x}(r, s, \mu)-x^{*} \\
\hat{w}(r, s, \mu)-\bar{w}^{*}
\end{array}\right]\right\| \leq \epsilon,
$$


for all $(r, s, \mu) \in B(K, \delta)$. Using (25c) and the strict complementarity of the pair $\left(x^{*}, \bar{w}^{*}\right)$, and reducing $\epsilon$ and $\delta$ if necessary, one can show easily that, for all $(r, s, \mu) \in B(K, \delta)$,

$$
\hat{x}(r, s, \mu) \geq 0, \quad \hat{w}(r, s, \mu) \geq 0 ;
$$

the pair $(\hat{x}(r, s, \mu), \hat{w}(r, s, \mu))$ verifies also strict complementarity; the gradients of the active constraints are linearly independent at $\hat{x}(r, s, \mu)$.

Step 5. Bounds (14). We differentiate (25) with respect to $(r, s, \mu)$, and write

$$
J(r, s, \mu)\left[\begin{array}{lll}
\nabla_{r} \hat{x}(r, s, \mu)^{\top} & \nabla_{s} \hat{x}(r, s, \mu)^{\top} & \nabla_{\mu} \hat{x}(r, s, \mu)^{\top} \\
\nabla_{r} \hat{w}(r, s, \mu)^{\top} & \nabla_{s} \hat{w}(r, s, \mu)^{\top} & \nabla_{\mu} \hat{w}(r, s, \mu)^{\top}
\end{array}\right]=-B(r, s, \mu) .
$$

Here, $J(r, s, \mu)$ is the Jacobian of the vector function of the left-hand side of (25) with respect to $x$ and $w$, given by

$$
\begin{aligned}
& {\left[\begin{array}{cc}
-\mu \nabla h^{\top} \nabla_{x x}^{2} l\left(I-Z Z^{\top}\right)+\nabla h^{\top} \nabla h \nabla h^{\top} & -\mu \nabla h^{\top} \\
Z^{\top} \nabla_{x x}^{2} l Z Z^{\top} & -Z^{\top} \\
\hat{W} & \hat{X}
\end{array}\right]} \\
& +\left[\begin{array}{ll}
A_{11}-\mu \nabla h^{\top} R^{\top}\left(\nabla h^{\top} \nabla h\right)^{-1} \nabla h^{\top}+\mu \nabla h^{\top}\left(\nabla_{x x}^{2} l \nabla h+R^{\top}\right) F(h) \\
-\mu \sum_{i=1}^{m} \nabla_{x}\left[\nabla h^{\top}\left(\nabla_{x x}^{2} l \nabla h+R^{\top}\right)\right]_{i}\left[\left(\nabla h^{\top} \nabla h\right)^{-1} h\right]_{i}+\sum_{i=1}^{m} h_{i} \nabla\left(\nabla h^{\top} \nabla h\right)_{i} & 0 \\
A_{21}-\sum_{i=1}^{m}\left[\left(\nabla h^{\top} \nabla h\right)^{-1} h\right]_{i} \nabla_{x}\left[Z^{\top}\left(\nabla_{x x}^{2} l \nabla h+R^{\top}\right)\right]_{i} & \\
+Z^{\top}\left(\nabla_{x x}^{2} l \nabla h+R^{\top}\right) F(h)-Z^{\top} R^{\top}\left(\nabla h^{\top} \nabla h\right)^{-1} \nabla h^{\top} & 0 \\
0 & 0
\end{array}\right],
\end{aligned}
$$

where the functions $h, Z, \nabla h, \nabla^{2} h_{i}, i=1, \ldots, m$, are evaluated at $\hat{x}(r, s, \mu)$ and the functions $\nabla_{x x}^{2} l$ and $R$ are evaluated at $(\hat{x}(r, s, \mu), \hat{w}(r, s, \mu))$, and where the rows of $A_{11}$ are given by

$$
\left(A_{11}\right)_{i}=\left[r+\mu\left(\bar{w}^{*}-\bar{w}\right)\right]^{\top} \nabla^{2} h_{i}, \quad i=1, \ldots, m .
$$

The term $A_{21}$ is given by

$$
\begin{aligned}
A_{21} & =\left[\begin{array}{c}
(\nabla f-\bar{w})^{\top} \nabla Z_{1}^{\top} \\
\vdots \\
(\nabla f-\bar{w})^{\top} \nabla Z_{n-m}^{\top}
\end{array}\right]+Z^{\top} \nabla^{2} f-Z^{\top} \nabla_{x x}^{2} l \\
& =\left[\begin{array}{c}
(\nabla f-\bar{w})^{\top} Z Z^{\top} \nabla Z_{1}^{\top} \\
\vdots \\
(\nabla f-\bar{w})^{\top} Z Z^{\top} \nabla Z_{n-m}^{\top}
\end{array}\right],
\end{aligned}
$$

where the last equality is justified by the derivation (22). 
In (30), $B$ is the Jacobian of the vector function on the left-hand side of (25) with respect to $r, s, \mu$, defined by

$$
B(r, s, \mu)=\left[\begin{array}{lll}
B_{11}(r, s, \mu) & B_{12}(r, s, \mu) & B_{13}(r, s, \mu) \\
0 & B_{22}(r, s, \mu) & 0 \\
0 & 0 & 0
\end{array}\right],
$$

with

$$
\begin{aligned}
B_{11}(r, s, \mu)= & \nabla h^{\top}, \\
B_{12}(r, s, \mu) e_{j}= & -\mu \nabla h^{\top} \sum_{i=1}^{m}\left[\left(\nabla h^{\top} \nabla h\right)^{-1} \nabla h^{\top} e_{j}\right]_{i} \nabla^{2} h_{i} \nabla h\left(\nabla h^{\top} \nabla h\right)^{-1} h \\
& +\mu \nabla h^{\top}\left[\begin{array}{c}
\left(Z Z^{\top} e_{j}\right)^{\top} \nabla^{2} h_{1} \\
\vdots \\
\left(Z Z^{\top} e_{j}\right)^{\top} \nabla^{2} h_{m}
\end{array}\right]\left(\nabla h^{\top} \nabla h\right)^{-1} h, \\
B_{13}(r, s, \mu)=- & \nabla h^{\top}\left[\left(\nabla_{x x}^{2} l \nabla h+R^{\top}\right)\left(\nabla h^{\top} \nabla h\right)^{-1} h+\bar{w}^{*}-\hat{w}(r, s, \mu)\right], \\
B_{22}(r, s, \mu) e_{j}= & -Z^{\top} \sum_{i=1}^{m}\left[\left(\nabla h^{\top} \nabla h\right)^{-1} \nabla h^{\top} e_{j}\right]_{i} \nabla^{2} h_{i} \nabla h\left(\nabla h^{\top} \nabla h\right)^{-1} h \\
& +Z^{\top}\left[\begin{array}{c}
\left(Z Z^{\top} e_{j}\right)^{\top} \nabla^{2} h_{1} \\
\vdots \\
\left(Z Z^{\top} e_{j}\right)^{\top} \nabla^{2} h_{m}
\end{array}\right]\left(\nabla h^{\top} \nabla h\right)^{-1} h,
\end{aligned}
$$

where $j=1, \ldots, n$.

Hence, for all $(r, s, \mu) \in B(K, \delta)$, we have

$$
\begin{aligned}
{\left[\begin{array}{l}
\hat{x}(r, s, \mu)-x^{*} \\
\hat{w}(r, s, \mu)-\bar{w}^{*}
\end{array}\right] } & =\left[\begin{array}{l}
\hat{x}(r, s, \mu)-\hat{x}(0,0,0) \\
\hat{w}(r, s, \mu)-\hat{w}(0,0,0)
\end{array}\right] \\
& =-\int_{0}^{1} J(\tau r, \tau s, \tau \mu)^{-1} B(\tau r, \tau s, \tau \mu)\left(r^{\top} s^{\top} \mu\right)^{\top} d \tau .
\end{aligned}
$$

Since $J^{*}(0,0, \mu)$ is nonsingular for all $\mu \in\left[0, \mu^{*}\right]$, we can show that, for $\epsilon$ and $\delta$ sufficiently small, $J(r, s, \mu)^{-1}$ is bounded on

$$
\left\{(r, s, \mu):\|(r, s)\|<\delta, \mu \in\left[0, \mu^{*}\right]\right\} \subset B(K, \delta) .
$$

In fact, it is quite clear from the continuity assumptions that the first matrix term of $J(r, s, \mu)$ is a perturbation of size $\delta$ and $\epsilon$ of $J^{*}(0,0,0)$. If we look carefully at the second term of $J(r, s, \mu)$, we come to the conclusion that all the expressions involved depend continuously on either $r, \mu, h, R$ or $Z^{\top}(\nabla f-\bar{w})$, quantities that are of size $\delta$ and $\epsilon$. 
Now, we can show finally (14). By appealing to

$$
\begin{aligned}
& \left\|\left[\begin{array}{l}
\hat{x}(r, s, \mu)-x^{*} \\
\hat{w}(r, s, \mu)-\bar{w}^{*}
\end{array}\right]\right\| \\
& \leq \max _{\tau \in[0,1]}\left\|J(\tau r, \tau s, \tau \mu)^{-1}\right\| \int_{0}^{1}\left\|B(\tau r, \tau s, \tau \mu)\left(r^{\top} s^{\top} \mu\right)^{\top}\right\| d \tau,
\end{aligned}
$$

and by applying the continuity assumptions to the terms that appear in $B_{11}(r, s, \mu), B_{12}(r, s, \mu), B_{13}(r, s, \mu), B_{22}(r, s, \mu)$, we can assume the existence of positive constants $\kappa_{3}$ to $\kappa_{7}$ such that

$$
\begin{aligned}
& \left\|\hat{x}(r, s, \mu)-x^{*}\right\|+\left\|\hat{w}(r, s, \mu)-\bar{w}^{*}\right\| \\
& \leq \kappa_{3}\|r\|+\kappa_{4} \mu\|s\| d(r, s, \mu)+\kappa_{5} \mu d(r, s, \mu) \\
& \quad+\kappa_{6} \mu \max _{\tau \in[0,1]}\left\|\hat{w}(\tau r, \tau s, \tau \mu)-\bar{w}^{*}\right\|+\kappa_{7}\|s\| d(r, s, \mu),
\end{aligned}
$$

where

$$
d(r, s, \mu)=\max _{\tau \in[0,1]}\left\|\left\{\nabla h(\hat{x}(\tau r, \tau s, \tau \mu))^{\top} \nabla h(\hat{x}(\tau r, \tau s, \tau \mu))\right\}^{-1} h(\hat{x}(\tau r, \tau s, \tau \mu))\right\| .
$$

Furthermore, from (25a) we write, with $\hat{x}=\hat{x}(r, s, \mu)$ and $\hat{w}=\hat{w}(r, s, \mu)$,

$$
\begin{aligned}
h(\hat{x})= & \nabla h(\hat{x})^{\top} \nabla h(\hat{x})\left\{-\mu \nabla h(\hat{x})^{\top}\left[\nabla_{x x}^{2} l(\hat{x}, \hat{w}) \nabla h(\hat{x})+R(\hat{x}, \hat{w})^{\top}\right]\right. \\
& \left.+\nabla h(\hat{x})^{\top} \nabla h(\hat{x}) \nabla h(\hat{x})^{\top} \nabla h(\hat{x})\right\}^{-1} \nabla h(\hat{x})^{\top}\left(-r+\mu \hat{w}-\mu \bar{w}^{*}\right) .
\end{aligned}
$$

Thus, the choice of $\mu^{*}$ and the continuity assumptions, together with the expression (31) for $h(\hat{x})$, imply that

$$
d(r, s, \mu) \leq \kappa_{8}\|r\|+\kappa_{9} \mu \max _{\tau \in[0,1]}\left\|\hat{w}(\tau r, \tau s, \tau \mu)-\bar{w}^{*}\right\|,
$$

for some positive constants $\kappa_{8}$ and $\kappa_{9}$. Since $\mu \leq \mu^{*}$ and $\|s\|<\delta$, there exist positive constants $\kappa_{10}$ and $\kappa_{11}$ such that

$$
\begin{aligned}
& \left\|\hat{x}(r, s, \mu)-x^{*}\right\|+\left\|\hat{w}(r, s, \mu)-\bar{w}^{*}\right\| \\
& \leq \kappa_{10}\|r\|+\kappa_{11} \mu \max _{\tau \in[0,1]}\left\|\hat{w}(\tau r, \tau s, \tau \mu)-\bar{w}^{*}\right\|,
\end{aligned}
$$

from which we get, for $(r, s, \mu)$ replaced by $(\tau r, \tau s, \tau \mu)$,

$$
\max _{\tau \in[0,1]}\left\|\hat{w}(\tau r, \tau s, \tau \mu)-\bar{w}^{*}\right\| \leq\left[\kappa_{10} /\left(1-\kappa_{11} \mu\right)\right]\|r\|,
$$

for $\mu \in[0, \bar{\mu}]$, with $\mu<\min \left\{\mu^{*}, 1 / \kappa_{11}\right\}$. Therefore,

$$
\begin{aligned}
& \left\|\hat{x}(r, s, \mu)-x^{*}\right\|+\left\|\hat{w}(r, s, \mu)-\bar{w}^{*}\right\| \\
& \leq\left[\kappa_{10}+\kappa_{10} \kappa_{11} \mu /\left(1-\kappa_{11} \mu\right)\right]\|r\| \\
& \leq\left[\kappa_{10} /\left(1-\kappa_{11} \bar{\mu}\right)\right] \mu\left\|w-\bar{w}^{*}\right\| .
\end{aligned}
$$


For $\mu \in\left(0, \mu^{*}\right]$ and $\left\|w-\bar{w}^{*}\right\|<\min \{\delta, \delta / \mu\}$, let us define

$$
\begin{aligned}
& \tilde{x}(w, \mu)=\hat{x}(r, s, \mu)=\hat{x}\left(\mu\left(w-\bar{w}^{*}\right), w-\bar{w}^{*}, \mu\right), \\
& \tilde{w}(w, \mu)=\hat{w}(r, s, \mu)=\hat{w}\left(\mu\left(w-\bar{w}^{*}\right), w-\bar{w}^{*}, \mu\right) .
\end{aligned}
$$

Hence, the bounds (14) follow immediately from (32).

Step 6. Optimality of $\tilde{x}(w, \mu)$. We finish the proof by showing that $\tilde{x}(w, \mu)$ is the solution of problem (12). First, we point out that $(\tilde{x}(w, \mu), \tilde{w}(w, \mu))$ satisfies the first-order necessary conditions for (12) as it can be seen by rewriting the system (25) using the changes of variables (24) and (33). The first equation of the first-order necessary conditions is, with $\tilde{x}=\tilde{x}(w, \mu)$ and $\tilde{w}=\tilde{w}(w, \mu)$,

$$
\begin{aligned}
& Z(\tilde{x}) Z(\tilde{x})^{\top} \nabla f(\tilde{x})+\left[I-Z(\tilde{x}) Z(\tilde{x})^{\top}\right] w \\
& -\left[\nabla_{x x}^{2} l(\tilde{x}, w) \nabla h(\tilde{x})+R(\tilde{x}, w)^{\top}\right]\left[\nabla h(\tilde{x})^{\top} \nabla h(\tilde{x})\right]^{-1} h(\tilde{x}) \\
& +(1 / \mu) \nabla h(\tilde{x}) h(\tilde{x})-\tilde{w}=0,
\end{aligned}
$$

and (15) is clearly true. Now, we show that the Hessian of $P(x ; w, \mu)$ is positive definite at $\tilde{x}(w, \mu)$ for all vectors

$$
(\Delta x)_{i}=0, \quad \text { if }(\tilde{x}(w, \mu))_{i}=0 \text { and }(\tilde{w}(w, \mu))_{i}>0 .
$$

The case $(\Delta x)_{i} \geq 0$ is eliminated, because the pair $(\tilde{x}(w, \mu), \tilde{w}(w, \mu))$ is strictly complementary. The scalar $\epsilon$ can be chosen sufficiently small so that we can consider

$$
(\Delta x)_{i}=0, \quad \text { if } x_{i}^{*}=0 \text { and }\left(\bar{w}^{*}\right)_{i}>0 .
$$

This means that we can check the positive definiteness of the Hessian of $P(x ; w, \mu)$ in the same subspace that we consider for $P\left(x^{*} ; \bar{w}^{*}, \mu\right)$. Moreover, we proved in Theorem 3.1 that the Hessian of $P\left(x^{*} ; \bar{w}^{*}, \mu\right)$ is positive definite for $\mu \in\left(0, \mu^{*}\right]$ in the above mentioned subspace. To achieve our goal, we show that the Hessian of $P(\tilde{x}(w, \mu) ; w, \mu)$ is a perturbation of size $\epsilon$ and $\delta$ of the Hessian of $P\left(x^{*} ; \bar{w}^{*}, \mu\right)$. In fact, the Hessian of $P(\tilde{x}(w, \mu) ; w, \mu)$ is given by

$$
\begin{aligned}
& \nabla_{x x}^{2} l Z Z^{\top}-R^{\top}\left(\nabla h^{\top} \nabla h\right)^{-1} \nabla h^{\top}-\left(I-Z Z^{\top}\right) \nabla_{x x}^{2} l-\nabla h\left(\nabla h^{\top} \nabla h\right)^{-1} R \\
& +\sum_{i=1}^{m} h_{i} \nabla_{x x}^{2} \lambda_{i}+(1 / \mu) \nabla h \nabla h^{\top}+(1 / \mu) \sum_{i=1}^{m} h_{i} \nabla^{2} h_{i},
\end{aligned}
$$

see (10), with the Lagrangian and the residual $R$ evaluated at $(\tilde{x}(w, \mu), w)$ and the remaining functions at $\tilde{x}(w, \mu)$. The term

$$
\begin{aligned}
& \nabla_{x x}^{2} l Z Z^{\top}-R^{\top}\left(\nabla h^{\top} \nabla h\right)^{-1} \nabla h^{\top}-\left(I-Z Z^{\top}\right) \nabla_{x x}^{2} l \\
& -\nabla h\left(\nabla h^{\top} \nabla h\right)^{-1} R+(1 / \mu) \nabla h \nabla h^{\top}
\end{aligned}
$$


is a perturbation of size $\epsilon$ and $\delta$ of the Hessian of $P\left(x^{*} ; \bar{w}^{*}, \mu\right)$. To bound the remaining terms, we can rewrite (34), using $\tilde{x}=\tilde{x}(w, \mu)$ and $\tilde{w}=\tilde{w}(w, \mu)$, as

$$
\begin{aligned}
(1 / \mu) h(\tilde{x})= & \nabla h(\tilde{x})^{\top} \nabla h(\tilde{x})\left\{-\mu \nabla h(\tilde{x})^{\top}\left[\nabla_{x x}^{2} l(\tilde{x}, w) \nabla h(\tilde{x})+R(\tilde{x}, w)^{\top}\right]\right. \\
& \left.+\left[I-Z(\tilde{x}) Z(\tilde{x})^{\top}\right]\right\}^{-1} \nabla h(\tilde{x})^{\top}(\tilde{w}-w) .
\end{aligned}
$$

Thus, using the continuity assumptions and adding and subtracting $\bar{w}^{*}$, we obtain, for some positive constant $\kappa_{12}$,

$$
\begin{gathered}
\|(1 / \mu) h(\tilde{x}(w, \mu))\| \leq \kappa_{12}\left(\left\|\tilde{w}(w, \mu)-\bar{w}^{*}\right\|+\left\|w-\bar{w}^{*}\right\|\right) \\
\quad \leq \kappa_{12}(\epsilon+\delta), \\
\|h(\tilde{x}(w, \mu))\| \leq \bar{\mu} \kappa_{12}(\epsilon+\delta) .
\end{gathered}
$$

The conclusion is that

$$
\sum_{i=1}^{m} h_{i} \nabla_{x x}^{2} \lambda_{i}+(1 / \mu) \sum_{i=1}^{m} h_{i} \nabla^{2} h_{i}
$$

is also of size $\delta$ and $\epsilon$, and the proof that the Hessian of $P(x ; w, \mu)$ is positive definite for all vectors $\Delta x$ satisfying (35) is terminated.

The proof shows also that $\kappa_{1}$ and $\kappa_{2}$ in the bounds (14) grow with the condition number of $\nabla h^{\top} \nabla h$.

\section{References}

1. Bertsekas, D. P., Constrained Optimization and Lagrange Multiplier Methods, Computer Science and Applied Mathematics, Academic Press, New York, NY, 1982.

2. Conn, A. R., Gould, N. I. M., and Toint, P. L., A Globally Convergent Augmented Lagrangian Algorithm for Optimization with General Constraints and Simple Bounds, SIAM Journal on Numerical Analysis, Vol. 28, pp. 545-572, 1991.

3. Vicente, L. N., Local Analysis of a New Multipliers Method, European Journal of Operational Research, Vol. 143, pp. 432-451, 2002.

4. Dennis, J. E., Heinkenschloss, M., and Vicente, L. N., Trust-Region Interior-Point SQP Algorithms for a Class of Nonlinear Programming Problems, SIAM Journal on Control and Optimization, Vol. 36, pp. 1750-1794, 1998.

5. Conn, A. R., Gould, N. I. M., and Toint, P. L., LANCELOT: A Fortran Package for Large-Scale Nonlinear Optimization (Release A), Springer Verlag, New York, NY, 1992.

6. Goodman, J., Newton's Method for Constrained Optimization, Mathematical Programming, Vol. 33, pp. 162-171, 1985. 